American Journal of Applied Sciences 2 (4): 857-859, 2005

ISSN 1546-9239

(C) Science Publications, 2005

\title{
Localization of Implanted Impurities in Silicon
}

\author{
M. Jadan and Jihad S.M. Addasi \\ Department of Basic Sciences, Tafila Applied University College \\ Al-Balqa Applied University, P.O. Box 40, AL-Eys-66141, Tafila, Jordan
}

\begin{abstract}
Localization of implanted boron impurities at the nodes and interstitial of silicon depending on the implantation current density has been studied by the X-ray diffraction and astrophysical methods. A shares of the impurity at the lattice sites increases with growing current density due to the instantaneous vacancy concentration and suppression of the impurity displacement from the sites by silicon interstitial.
\end{abstract}

Keywords: Localization, silicon, impurities

\section{INTRODUCTION}

Localization of implanted impurities at the lattice is determined by its interaction with point radiationinduced defects. The impurity-defect interaction is one of the major problems associated with the physics of a real crystal. At the same time, a practical significance of this problem stems from the fact that modeling of the processes inherent in the high-temperature diffusion of the implanted impurity is based on the initial distribution of this impurity at the lattice sites and interstices.

Localization of the implanted impurities is as yet not clearly understood in spite of numerous works devoted to this problem. There is a significant divergence in the opinions of various authors as to the quantity of boron at silicon lattice sites immediately following the implantation at room temperature, the data varying from $20 \%$ to nearly $100 \%$ localization of boron at the sites. In case of the phosphorus its share of the lattice sites is as a whole greater than that of boron.

However, the results are also differing from work to other ${ }^{[1-5]}$. Moreover, the cause of this disparity remains unclarified. We believe that this discrepancy may be attributed to the differences in the implantation conditions. This paper studies the effect exerted by the implantation current density on localization of the implanted boron impurity in silicon.

Theory and Experimental Method: Investigations of boron implantation have been conducted for n-type silicon with a starting resistivity of $0.5 \Omega \cdot \mathrm{cm}$ and the silicon heavily doped with boron in the process of growth $\left(\rho_{0}=0.005 \Omega \cdot \mathrm{cm}\right)$. Ion-beam implantation (ion energy $100 \mathrm{keV}$ ) was performed at the effective density of ion current $\mathrm{j}_{\text {ef }}$ from 0.4 to $2 \mu \mathrm{A} \cdot \mathrm{cm}^{-2}$. X-ray diffraction studies have been realized using a twocrystal spectrometer with parallel arrangement of a crystal-monochromator and a sample under study at the $\mathrm{CuK}_{\mathrm{el}}$ emission line into $\mathrm{t}$ selection h-order plplaneslection from 111 planes.
The observed change in the lattice constant of silicon as a result of implantation is determined by two factors: by radiation defects causing an increase in lattice constant and by boron atoms which compress the lattice being positioned at the sites. This is due to the fact that the covalent radius of $\mathrm{B}$ atom $\left(0.8 \mathrm{~A}^{\circ}\right) \mathrm{Si}$ atoms than that of $\mathrm{Si}$ atoms $\left(1.175 \mathrm{~A}^{\circ}\right)$. Electric activation of the implanted impurities during isochronous annealing has been analyzed by measurements of Hall effect and conductivity using the Van-Der-Pauw method.

\section{RESULTS AND DISCUSSION}

Figure 1 shows the dependence of a change in lattice constant $(\Delta \alpha)$ in a silicon layer following the implantation of $\mathrm{B}$ ions with a dose of $1.8 \cdot 10^{15} \mathrm{~cm}^{-2}$ on the ion beam current density $\mathrm{j}_{\mathrm{ef}}$. As seen in Fig. 1, a change in lattice constant $(\Delta \alpha)$ increases considerably as the effective ion current density $\left(\mathrm{j}_{\mathrm{ef}}\right)$ is growing from 0.2 to $1 \mu \mathrm{A} \cdot \mathrm{cm}^{-2}$ but then $\Delta \alpha\left(\mathrm{j}_{\mathrm{ef}}\right)$ reveals saturation for $\mathrm{j}_{\text {ef }}$ more than $1 \mu \mathrm{A} \cdot \mathrm{cm}^{-2}$. It should be noted that no marked changes in the temperature of samples have been observed for implantation within the indicated range of the effective ion current density.

Figure 2 illustrates curves 1, 2, 3 for lattice constant recovery in the process of asynchronous annealing of silicon implanted by $\mathrm{B}^{+}$ions with an ion current density $j_{\text {ef }}$ of values: $0.04 \mu \mathrm{A} \cdot \mathrm{cm}^{-2}$ (curve 1) and $2 \mu \mathrm{A} \cdot \mathrm{cm}^{-2}$ (curve 2). Curve 3 illustrates the lattice constant recovery in heavily boron-doped silicon $\left(\rho_{0}=\right.$ $0.005 \Omega \cdot \mathrm{cm}$ ) and within silicon implanted with boron ions $\left(\mathrm{B}^{+}\right)$with a current density $\left(\mathrm{j}_{\mathrm{ef}}\right)$ of $0.2 \mu \mathrm{A} \cdot \mathrm{cm}^{-2}$. The lattice constant recovery curves are affected by the "reverse" annealing stages at 120 and $480^{\circ} \mathrm{C}$ (curve 2) for high ion current density $\mathrm{j}_{\mathrm{ef}}=2 \mu \mathrm{A} \cdot \mathrm{cm}^{-2}$.

The electric activation was studied for boron implanted in silicon, where the charge carrier concentration in the layer $\mathrm{N}_{\mathrm{s}}(\mathrm{T})$ is illustrated as a function of the temperature $\mathrm{T}$ in Fig. 3. The dependence $\mathrm{N}_{\mathrm{s}}(\mathrm{T})$ was taken for isochronous annealing for $15 \mathrm{~min}$ Fig. 3 a curve 1. Curve 2 in Fig. 3 a is associated with 
$\mathrm{N}_{s}(\mathrm{~T})$ obtained in case of asynchronous annealing with simultaneous irradiation of the implanted layer of electrons with energy of $10 \mathrm{keV}$ and current density of 5 $\mu \mathrm{A} \cdot \mathrm{cm}^{-2}$. In this case (curve 1 in Fig. 3 a) there is no stage of "reverse" annealing characteristic for the electrical activation of boron in silicon.

A decrease of change in lattice constant $\Delta \alpha$ with increasing ion current density jef (Fig. 1) doesn't relate to a decrease in the introductory rate of stable radiation defects. As indicated above, no growth of the samples of temperature has been observed during implantation, which supported by the behavior of $\Delta \alpha\left(j_{\mathrm{ef}}\right)$ curve, Fig. 1. Should an increase in $j_{\text {ef }}$ leads to heating of the sample, the temperature should be growing over the whole range of $\mathrm{j}_{\mathrm{ef}}$, whereas $\Delta \alpha\left(\mathrm{j}_{\mathrm{ef}}\right)$ should not reach saturation. A decreased $\Delta \alpha$ may be due to the greater number of boron atoms located at the lattice sites with the growing density of the ion beam. This in turn is determined by two factors: capture of boron atoms at the vacancy and its substitution at the lattice sites by interstitial silicon atoms arising from implantation (Watkins effect). An instantaneous vacancy concentration is growing with the density of ion current $\mathrm{j}_{\mathrm{ef}}$ facilitating transfer of boron to the lattice sites. The results obtained on isochronous annealing of the implanted silicon point to an increased number of boron atoms at the lattice sites with the growing density of the implantation current (Fig. 2). The samples implanted by $\mathrm{B}^{+}$ions with an effective current density $\mathrm{j}_{\mathrm{ef}}$ of 0.04 $\mu \mathrm{A} \cdot \mathrm{cm}^{-2}$ exhibit lattice constant recovery in three stages. The first stage is associated with annealing of deficiencies in the main, at the second stage primarily the multi-vacancy complexes are annealed. Similar stages of lattice constant recovery are also demonstrated in silicon implanted by $\mathrm{Si}$ ions ${ }^{[6]}$. The annealing stage at $700-900^{\circ} \mathrm{C}$ is characteristic of silicon implanted only by boron ions. The data ${ }^{[6]}$ indicate that at this stage the complexes between deficiencies and boron atoms are annealed.

In silicon samples implanted with boron ions with high-density ion current $j_{\mathrm{ef}}=2 \mu \mathrm{A} \cdot \mathrm{cm}^{-2}$ the characteristic stages of "reverse" annealing (Fig. 2 curves 2) are associated with boron displacement from the lattice sites into interstices by interstitial $\mathrm{Si}$ atoms ${ }^{[7]}$. These free atoms of silicon are formed from decomposition of the interstitial complexes. Si-P6centers are annealed at $120^{\circ} \mathrm{C}$ and $\mathrm{Si}-\mathrm{B} 3$-centers are annealed at $480^{\circ} \mathrm{C}$. Besides, the "reverse" annealing stages are observed in crystals irradiated by $\mathrm{B}^{+}$ions with a current density $j_{\text {ef }}$ of $0.2 \mu \mathrm{A} \cdot \mathrm{cm}^{-2}$ (curve 3) provided the initial concentration of boron at the lattice sites of $\mathrm{Si}$ is high $\left(\rho_{0}=0.005 \Omega \cdot \mathrm{cm}\right.$, hole concentration of (3-4) $\left.110^{19} \mathrm{~cm}^{-3}\right)$.

A high level of inelastic loss is characteristic of the process of ion-implantation doping (especially for light ions). In silicon implanted with $\mathrm{B}^{+}$ions with energy of $100 \mathrm{keV}$ inelastic losses come to about $80 \%$ and in ionization level in the crystal is rising with the ion current density $\mathrm{j}_{\mathrm{ef}}$. At a high ionization level displacement of the substitutional impurities is suppressed. This is attested by the results obtained for the electrical activation of implanted boron (Fig. 3 a). It is known that the "reverse" annealing stage of the electric activation curve of boron is due to substitution of interstitial $\mathrm{Si}$ atoms formed upon annealing of interstitial complexes SI-B3 for boron at the lattice sites. An increased ionization level with crystal through electron irradiation with energy of $10 \mathrm{keV}$ and current density $\mathrm{j}_{\mathrm{ef}}=5 \mu \mathrm{A} \cdot \mathrm{cm}^{-2}$ results in suppression of Watkins substitution.

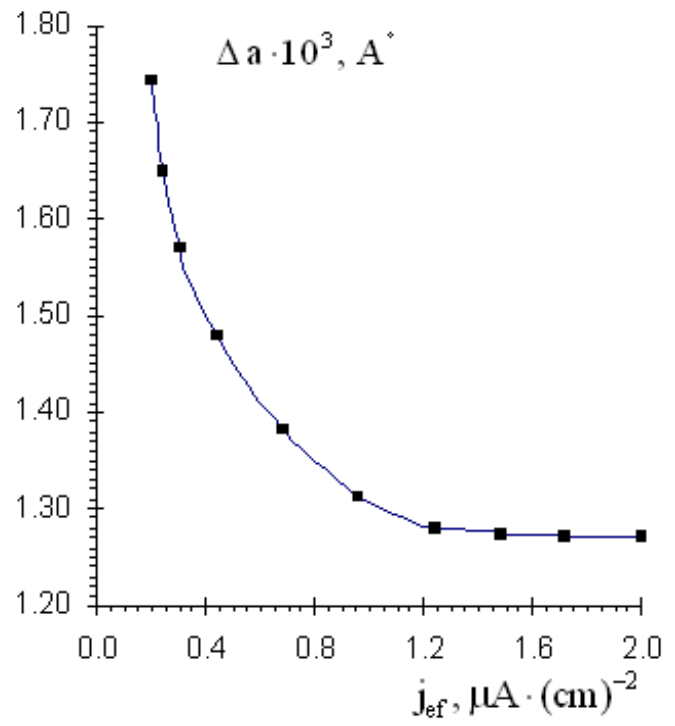

Fig. 1: Dependence of Change in Lattice Constant for Silicon $\Delta a$ on Boron Ion Current Density jef

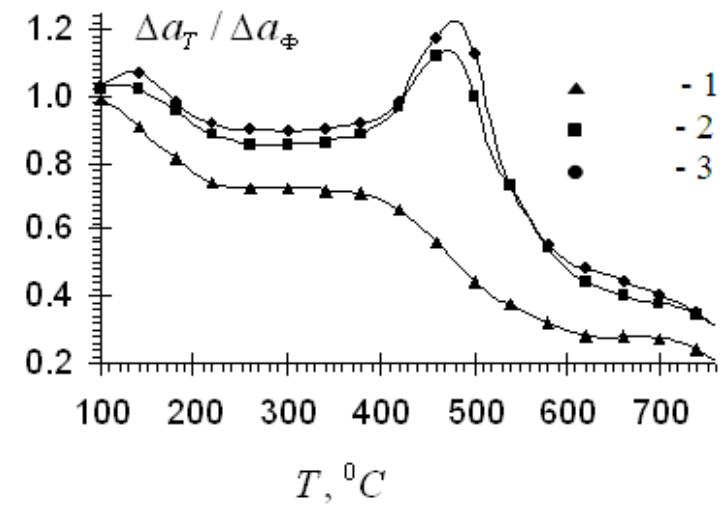

Fig. 2: Dependence of Lattice Constant $\Delta \alpha_{\mathrm{T}} / \Delta \alpha_{\Phi}$ in Si Irradiated by $\mathrm{B}^{+}$Ions on Temperature $\mathrm{T}$ for Curves: (1) Si: $\mathrm{P}, \rho_{0}=0.5 \Omega \cdot \mathrm{cm}, \mathrm{j}_{\mathrm{ef}}=0.04$ $\mu \mathrm{A} \cdot \mathrm{cm}^{-2}$; (2) Si: P, $\rho_{0}=0.5 \Omega \cdot \mathrm{cm}, \mathrm{j}_{\mathrm{ef}}=2$ $\mu \mathrm{A} \cdot \mathrm{cm}^{-2}$; and (3) Si: P, $\rho_{0}=0.5 \Omega \cdot \mathrm{cm}, \mathrm{j}_{\mathrm{ef}}=$ $0.2 \mu \mathrm{A} \cdot \mathrm{cm}^{-2}$ 

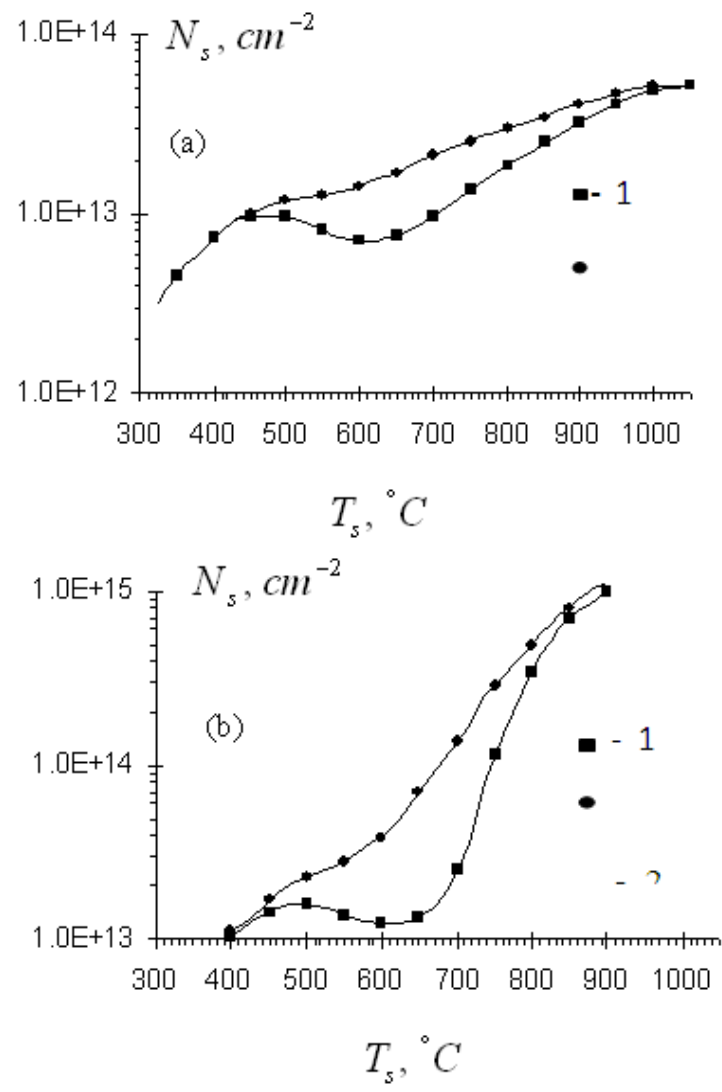

Fig. 3: Electric Activation of Implanted: (a) Boron Ions and (b) Phosphorus Ions in Silicon. This activation was realized for annealing (curve 1) without electron irradiation and (curve 2) with electron irradiation.

For a high dose of phosphorus (over $10^{14} \mathrm{~cm}^{-2}$ ) the implanted layer becomes amorphous. To observe the substitution of the interstitial $\mathrm{Si}$ atoms for phosphorus at the lattice sites (curve 1 Fig. 3 b), the silicon samples were annealed upon implantation by phosphorus with a dose of $1.5 \cdot 10^{15} \mathrm{~cm}^{-2}$ for crystallization of amorphous layer. After that the silicon samples annealed by $\mathrm{Si}^{+}$ ions with a dose of $10^{14} \mathrm{~cm}^{-2}$ to introduce radiation defects. In case of asynchronous annealing with simultaneous electron irradiation of the energy $10 \mathrm{keV}$ of the implanted layer no "reverse" annealing has been observed (curve 2 and Fig. 3b).

Interstitial $\mathrm{Si}$ atoms are moving to the deformation source (substitutional atom) in the field of elastic deformations created by this atom. From atomic point of view, this can be represented as follows. The lattice atoms displaced relative to the substitutional atom from the equilibrium positions in the first coordination sphere and progressively to a lesser degree at the subsequent spheres, become polarized and electric dipole is formed for each of them. Because of dipoledipole iteration, an interstitial atom of silicon is attracted to the polarized lattice atoms. Since the magnitude of a dipole moment at the displaced lattice atoms is drastically growing in the direction to the deformation source, the interstitial $\mathrm{Si}$ atom is moving to the substitution one. Should a high ionization level is created in the layer, non equilibrium electrons and holes are screening the dipoles at the displaced lattice atoms and the described migration mechanism becomes invalid. In this way, an elevated ionization level is liable to suppress the displacement of impurities from the lattice sites in the process of both implantation and subsequent annealing.

\section{CONCLUSION}

The results of research have demonstrated that the quantity of implanted impurity located at lattice sites immediately upon implantation at room temperature is determined by the implantation current density. This is governed by two factors. First, an instantaneous concentration of vacancy increases with the density of implantation current facilitating transfer of the impurity of the lattice sites. Second, the process of displacement of the substitutional impurities from the lattice sites by interstitial $\mathrm{Si}$ atoms is suppressed as the density of ion current is growing with associated increase in the concentration of non equilibrium charge carriers.

\section{REFERENCES}

1. North, J.C. and W.M. Gibson, 1970. Channeling study of boron-implanted silicon. Appl. Phys. Lett., 16: 126-129.

2. Fladda, G., K. Bjorkqvist, L. Eriksson and D. Siegard, 1970. The lattice location of boron ions implanted into silicon. Appl. Phys. Lett. 16: 313.

3. Eriksson, L., J.A. Davis, J. Denhartog, H. Matzke and J. L. Whitton, 1966. Can. Nucl. Technol., 5: 40-43.

4. Skakon, N.A., N.P. Diki, N.P. Mamash and P.A. Svemashiv, 1975. Phys. Of Heat and Cond., 9: 755.

5. Mayer, J.W., L. Eriksson and J.A. Davis, 1973. Ion implantation in semiconductors (Silicon and Germanium). Russ. Trans. by V.M. Guseva MIR Publishers Moscow, pp: 108.

6. Stelmakh, B.F., V.D. Tkachev and A.R. Chelyadinskii, 1978. X-ray diffraction. Studies of ion implanted boron in silicon. Phys. Solid St., 20: 2196.

7. Berezhnov, N.I., V.F. Stelmakh and A.R. Chelyadinskii, 1983. Interstitial type in ion implanted silicon: Phys. Status Solidi A., 78: 121.

8. Berezhnov, N.I., A.R. Chelyadinskii, M. Jadan and Yu. R. Suprun-belevich, 1993. On the problem of Watkins substitution and migration of silicon atoms in silicon. Nucl. Ins. Meth. B., 73: 357-361. 